\title{
México ante la epidemia de COVID-19 (SARS-CoV-2) y las recomendaciones al Subsistema Nacional de Donación y Trasplante
}

\section{Mexico's response to COVID-19 epidemic (SARS-CoV-2) and the recommendations to the National Donation and Transplant Subsystem}

\author{
José Salvador Aburto-Morales, ${ }^{*}$ Josefina Romero-Méndez, ${ }^{*}$ Cinthya Ayerim Lucio-García, ${ }^{*}$ \\ José André Madrigal-Bustamante, ${ }^{*}$ Grupo de expertos del Subsistema Nacional de Trasplantes ${ }^{\ddagger}$ \\ * Centro Nacional de Trasplantes. \\ ₹ Grupo de expertos conformado por: Aczel Sánchez Cedillo (Instituto de Seguridad y Servicios Sociales de los Trabaja- \\ dores del Estado), Érika Ribera Durón (Instituto Mexicano del Seguro Social), Josefina Alberú Gómez (Instituto Nacional \\ de Ciencias Médicas y Nutrición «Salvador Zubirán»), Nubia Denisse Avilez Pacheco (Instituto Mexicano del Seguro So- \\ cial), Rodrigo López Falcony (Centro Estatal de Trasplantes de Guanajuato y Sociedad Mexicana de Trasplantes).
}

\section{RESUMEN}

El Centro Nacional de Trasplantes, en coordinación con los integrantes del Subsistema Nacional de Trasplantes, emitió las «Recomendaciones al Subsistema Nacional de Donación y Trasplantes sobre la infección asociada al SARS-CoV-2 (COVID-19)», que orientan la actuación de los programas autorizados para procuración y trasplante en el país durante este periodo. Estas recomendaciones son producto de la revisión exhaustiva de la experiencia en países donde la pandemia inició antes que en México, de las principales publicaciones científicas publicadas sobre el tema y del trabajo del grupo de expertos mexicanos, con lo cual ha sido posible alinear las estrategias y acciones a nivel nacional, y reducir los riesgos y el impacto de esta emergencia sanitaria. Esto apoyará la preparación para las siguientes fases de la epidemia y permitirá tener información específica para la fase endémica; lo anterior con el objetivo de buscar el mayor beneficio y la seguridad de los receptores, donadores y del personal sanitario. En este documento se presentan los resultados del ejercicio de revisión, diseño e implementación de estas medidas.

Palabras clave: COVID-19, coronavirus, SARS-CoV-2, pandemias, trasplante, obtención de tejidos y órganos, México.

\section{ABSTRACT}

The National Transplant Center and members of the National Transplant Subsystem delivered «The Recommendations to the National Donation and Transplant Subsystem about the infection caused by SARS-CoV-2 (COVID-19)", which is a guide for national programs authorized for procurement and transplantation during the outbreak. These recommendations are based on the main scientific publications, the suggestions from national experts and the results of the comprehensive reviews from countries previously affected by the pandemic. It has resulted in the possibility to align strategies and actions at the national level to reduce the risks and impact of this health emergency. This will support the preparedness for the next phases of the epidemic and will allow having specific information for the endemic phase. The aim is to seek out the greatest benefit and safety for recipients, donors and healthcare workers. This document presents the results of the revision, design and implementation of The Recommendations.

Keywords: COVID-19, coronavirus, SARS-CoV-2, pandemics, transplantation, tissue and organ procurement, Mexico. 


\section{INTRODUCCIÓN}

La pandemia por COVID-19 tiene su antecedente en Wuhan, provincia de Hubei, China, donde se reportaron casos de pacientes con neumonía de causa desconocida vinculada principalmente a trabajadores de un mercado mayorista de mariscos y animales silvestres. Posteriormente, se pudo identificar que se trataba de un nuevo virus, del tipo coronavirus, que se supone saltó del murciélago a un mamífero intermediario (se sospecha del pangolín), y de ahí a los humanos.

El virus SARS-CoV-2 es un betacoronavirus, como el MERS-CoV (síndrome respiratorio de Oriente Medio) y el SARS-CoV (síndrome respiratorio agudo grave). La vía de trasmisión es a través de pequeñas gotas de saliva emitidas por una persona infectada al estornudar, toser o al tocarse los ojos, nariz o boca después de tener contacto con superficies contaminadas. Se ha reportado que el virus puede permanecer viable por horas en superficies de cobre, o hasta por uno o dos días en plástico o acero. ${ }^{1}$

La persona infectada puede trasmitir el virus incluso antes de la aparición de la sintomatología y se sabe que un número importante de casos pueden ser asintomáticos y ser trasmisores. Adicionalmente, la Organización Mundial de la Salud (OMS), basada en el comportamiento de casos en China, ha estimado alta contagiosidad del SARS-CoV-2, con una tasa de contagio (R0) del virus entre 1.4 a 2.5. Otras estimaciones indican un rango entre dos y tres, aunque se ha visto que puede haber personas capaces de contagiar hasta a 16 personas. Para controlar una epidemia, la R0 necesita disminuir por debajo de uno. ${ }^{2}$

Aunque se ha avanzado en el conocimiento de esta patología, aún existen vacíos de conocimiento sobre la historia natural. Hoy se conoce que se han presentado casos que van desde los asintomáticos, los que presentan un cuadro leve ( $80 \%$ ) hasta uno grave (16\%), y aquellos con un desenlace fatal. La sintomatología se caracteriza por dolor de garganta, tos, fiebre, cefalea y dificultad para respirar (en casos graves); en menos casos se ha identificado también la presencia de diarrea.

Los pacientes considerados como grupos de riesgo son los adultos mayores de 65 años, pacientes de cualquier edad con enfermedades crónicas cardiovasculares, hipertensión arterial, diabetes, enfermedad pulmonar, cáncer, enfermedad renal crónica y aquellos inmunocomprometidos o con tratamiento inmunosupresor, como los pacientes receptores de trasplante. Un reporte semanal de morbilidad y mortalidad $(\mathrm{MMWR})^{3}$ de los CDC (Center for Disease Control) analizó la gravedad de los casos registrados en Estados Unidos: por grupo de edad reveló que $80 \%$ de los casos de muertes correspondía a adultos de mayores de 65 años de edad y que en aquellos de más de 85 se incrementaba de manera importante.

El avance de la epidemia en el mundo llevó a la OMS a declarar a la nueva infección por coronavirus (COVID-19) como una pandemia el 11 de marzo de 2020. Las pandemias siguen una determinada progresión que comienza con una fase de investigación, seguida por fases de reconocimiento, iniciación y ace-

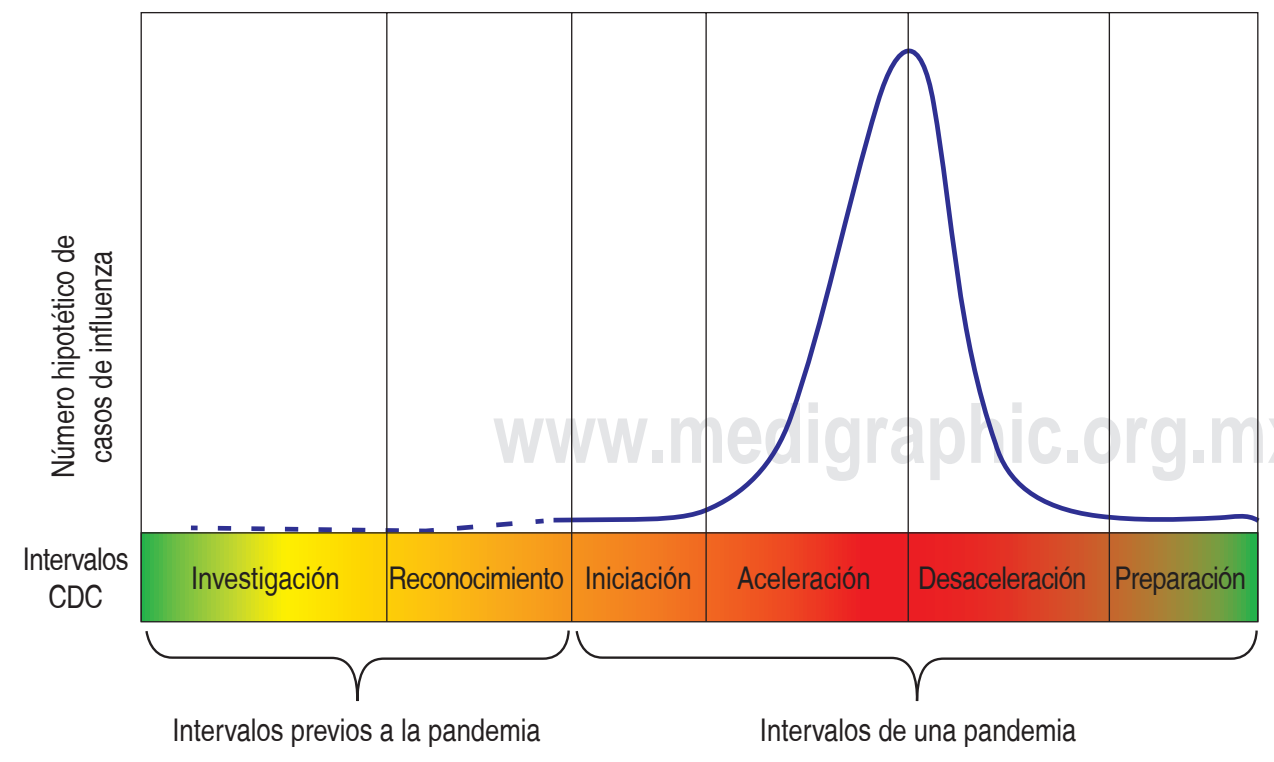

Figura 1:

Fases de una pandemia. Fuente: Centros para el Control y la Prevención de Enfermedades CN de V y ER (NCIRD). Marco de intervalos de una pandemia (PIF). Influenza pandémica (influenza). CDC. Published 2016. [Accessed April 17, 2020] Disponible en: https://espanol.cdc.gov/ flu/pandemic-resources/national-strategy/ intervals-framework.html. 
leración. Como se muestra en la Figura 1, los países pueden encontrarse en diferentes fases de la pandemia en cualquier momento dado, y diferentes partes de un mismo país también pueden estar atravesando fases distintas de la misma. ${ }^{4}$

Hasta el momento no existe tratamiento farmacológico específico y la posibilidad de una vacuna no se vislumbra cercana, hasta un año o año y medio. En ausencia de vacunas o medicamentos específicos para su tratamiento, las estrategias de salud pública son la respuesta más importante. Se trata de intervenciones en la comunidad que pueden reducir el riesgo e impacto de la enfermedad, en la población y en la sobrecarga de los recursos disponibles en los sistemas de salud.

Desde la aparición de los primeros casos en China al 16 de abril, son ya 212 países que reportan infectados con COVID-19 en diferentes fases de avance de la epidemia, con un total de casos confirmados de $2,088,425$, muertes 139,419 ; y personas evaluadas $3,262,921$. La tasa de letalidad global acumulada fue de $6.4 \%$. A nivel regional, las cifras reportadas por la OMS de casos acumulados muestran el mayor número en Europa, con 977,596; seguido de las Américas con 673,361; Mediterráneo Oriental con 107,389; Asia Sudoriental con 20,287; Pacífico Occidental con 124,916 y África con $11,367 .{ }^{5}$ Los países que concentran el mayor número de casos confirmados se muestran en la Tabla 1.

Con base en las primeras comunicaciones de la nueva enfermedad de Wuhan, México alertó y ha puesto en práctica actividades de preparación, que permitan hacer frente a las diferentes fases de la epidemia: 1) importación viral, en donde se espera detec-

Tabla 1: Número de casos confirmados y muertes por país, 2020.

\begin{tabular}{lcc}
\hline \multicolumn{1}{c}{ País } & No. casos confirmados & No. de muertes \\
\hline Estados Unidos & 640,014 & 26,059 \\
España & 182,816 & 19,130 \\
Italia & 165,155 & 21,645 \\
Alemania & 135,549 & 3,850 \\
Francia & 134,598 & 17,167 \\
Reino Unido & 104,133 & 13,729 \\
China & 83,402 & 3,346 \\
Irán & 77,995 & 4,869 \\
Turquía & 69,392 & 1,518 \\
Bélgica & 34,809 & 4,857 \\
\hline
\end{tabular}

Fuente: COVID-19 Dashboard by the Center for Systems Science and Engineering (CSS). https://gisanddata.maps.arcgis.com/apps/opsdashboard/index.htm|\#/ bda7594740fd40299423467b48e9ecf6 tar los primeros casos provenientes de los países con transmisión local sostenida; 2) dispersión comunitaria, una vez importado el virus a México, se espera la presencia de brotes comunitarios, y 3 ) fase de epidemia, con presencia del virus en todo el territorio y de brotes de COVID-19 en varias regiones del país. ${ }^{6}$

El primer caso confirmado se presentó en la Ciudad de México el 28 de febrero, posteriormente se confirmaron otros dos casos, en Sinaloa y en Ciudad de México, todos considerados como importados. El primer fallecimiento por COVID-19 ocurrió el 18 de marzo de 2020, y se trató de un mexicano con antecedente de haber viajado a Italia.

El Gobierno de México, en coordinación con la Secretaría de Salud, ha implementado medidas para prevenir y controlar la epidemia. A partir del 23 de marzo se informó de la extensión del periodo vacacional estudiantil y la Jornada Nacional de Sana Distancia, que incluye el distanciamiento social; lavado frecuente de manos; etiqueta respiratoria de saludo a distancia; Campaña "Quédate en Casa» y aislamiento en casa de los casos sospechosos o confirmados de COVID-19.

El 24 de marzo se decretó la fase 2, que incluye la suspensión de actividades «no esenciales», aquellas que no afectan la operación primordial de empresas, organizaciones y de gobierno, así como las que congreguen a personas o que impliquen que haya desplazamientos constantes en el transporte público. Especial cuidado y recomendación para quedarse en casa en los grupos considerados de mayor riesgo, como adultos mayores y aquellos que tienen alguna patología crónica como diabetes, hipertensión, pacientes inmunocomprometidos, obesidad, etc.

Posteriormente, el 30 de marzo, en reunión del Consejo de Salubridad General, se declaró una "emergencia sanitaria por causa de fuerza mayor»" en consideración a la evolución de la morbilidad y mortalidad relacionadas al SARS-COV-2, con lo cual se sustentan las acciones implementadas en la fase 2. Ese mismo día se anunció que la Marina y el Ejército se sumaban con el Plan DN-III para brindar apoyo ante la declaratoria de Decreto Presidencial por la emergencia sanitaria por el COVID-19.

Como se observa en la Figura 2, en México, al día 15 de abril se han confirmado 5,847 casos y 449 defunciones por COVID-19, con una tasa de letalidad de $7.5 \%$. En la Tabla 2 se muestran las entidades federativas donde se concentra el mayor número de casos confirmados de COVID-19, destacando la Ciudad de México, el Estado de México, Baja California, Sinaloa, Sonora, Puebla, Quintana Roo, Nuevo León, Tabasco, Coahuila y Jalisco. 


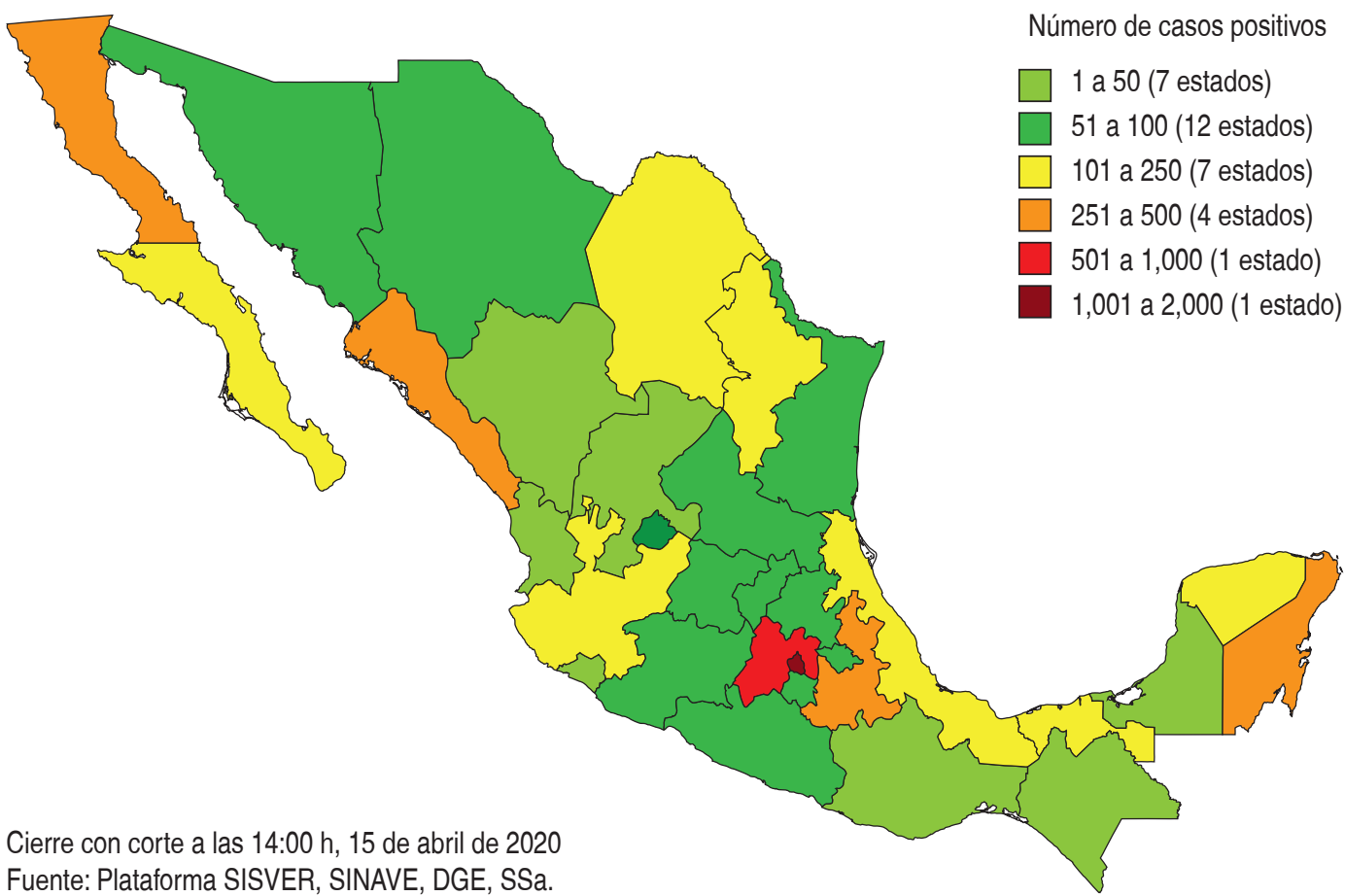

Figura 2: Casos confirmados, negativos y sospechosos a COVID-19. México 15 abril 2020.

Fuente: Secretaría de Salud. Informe técnico diario COVID-19. 15 de abril de 2020 (corte 14:00 h).

El grupo de edad más afectado por la infección por COVID-19 se encuentra entre los 40 y 49 años (22.1\%), seguido por los de $30-39(20.8 \%)$ y 50 a 59 años (19.1\%). Sin embargo, la mortalidad es mayor en la población de más de 65 años.

Este panorama y la consecuente necesidad de emprender acciones en materia de donación y trasplante de órganos y tejidos derivaron en el documento que a continuación se presenta. Éste contiene los resultados del ejercicio de revisión, diseño e implementación de medidas para hacer frente a la pandemia por COVID-19 en la población objetivo.

\section{LA DEFINICIÓN DE LAS RECOMENDACIONES}

En torno a la necesidad de contar con orientación para atender los aspectos relacionados con la infección por SARS-CoV-2 y el impacto generado en la actividad de donación y trasplantes en México,' se elaboraron las recomendaciones mediante un grupo de consenso. El documento está dirigido principalmente a coordinado-

El nuevo coronavirus se llama SARS-CoV-2. La enfermedad que causa se conoce como COVID-19 res hospitalarios de donación, programas de donación y trasplante de órganos y tejidos, centros y consejos estatales de trasplante y coordinaciones institucionales de donación y trasplantes.

Las recomendaciones se basan en las guías de la Organización Nacional de Trasplantes de España (ONT), la Sociedad Latinoamericana de Nefrología e Hipertensión (SLANH), Sociedad de Trasplantes de América Latina y el Caribe (STALYC), Asociación Panamericana de Infectología (API), así como las emitidas por el Instituto Nacional Central Único Coordinador de Ablación e Implante de Argentina (INCUCAI). Asimismo, se incorporaron recomendaciones consideradas pertinentes por el grupo de expertos del Instituto Mexicano del Seguro Social (IMSS), el Instituto de Seguridad y Servicios Sociales de los Trabajadores del Estado (ISSSTE), Instituto Nacional de Ciencias Médicas y Nutrición «Salvador Zubirán» y la Sociedad Mexicana de Trasplantes (SMT). El documento fue sometido a revisión por diferentes áreas de la Secretaría de Salud, como la Dirección General de Epidemiología y la Oficina de la Abogada General. Algunos puntos podrían requerir actualizaciones adicionales de acuerdo con las fases y la evolución del conocimiento sobre la epidemia. 
Tabla 2: Casos de coronavirus, México, 16 de abril de 2020.

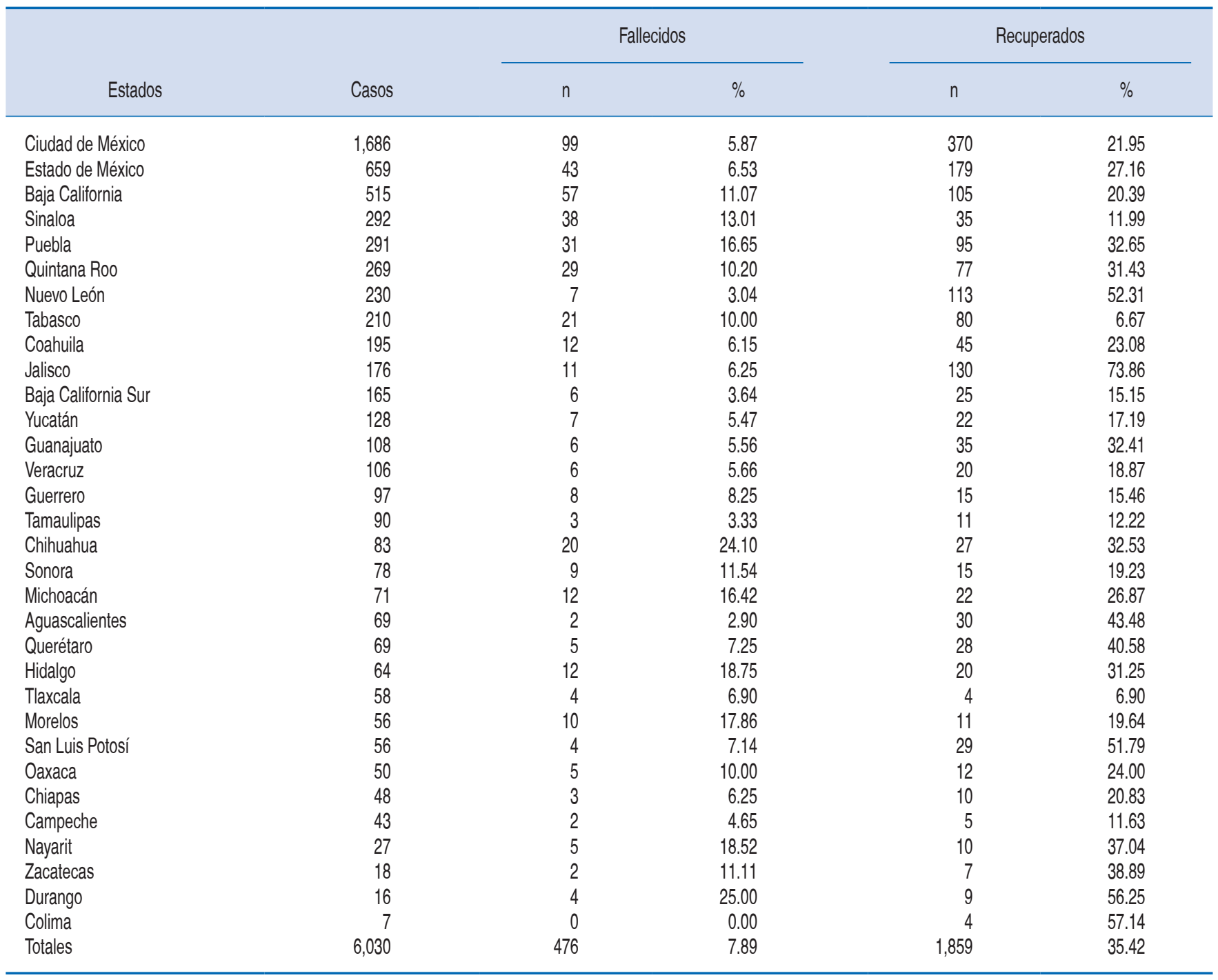

Fuentes: Secretaría de Salud (2020) e Instituto de Investigaciones Geológicas y Atmosféricas (2020).

\section{Consideraciones generales}

Hasta la fecha se han documentado pocos casos sobre receptores de trasplantes de órgano sólido infectados por SARS-CoV-2. . $^{-12}$

Si bien se desconoce el potencial de transmisión del SARS-CoV-2 a través del trasplante de órganos, tejidos o células humanas, existe un riesgo teórico y se deberán aplicar medidas de precaución a los receptores y profesionales de la salud. Algunos autores reconocen que el COVID-19 puede presentar un riesgo por la propagación nosocomial para los equipos de procuración y trasplante, pacientes y otros trabajado- res de la salud, basado en lo ocurrido con el SARSCoV 2002. ${ }^{13}$

En el documento se utilizan las definiciones operacionales de caso sospechoso y confirmado de COVID-19, de acuerdo con lo dispuesto por el Comité Nacional para la Vigilancia Epidemiológica (CONAVE). Están sujetas a cambios y serán actualizadas vía oficio y página web del CENATRA."

Se define como caso sospechoso a la persona de cualquier edad que en los últimos siete días haya presentado al menos dos de los siguientes signos y sínto-

ii https://www.gob.mx/cenatra 
mas: tos, fiebre o cefalea, iii acompañados de al menos uno de los siguientes signos o síntomas: disnea (dato de gravedad), artralgias, mialgias, odinofagia, rinorrea, conjuntivitis o dolor torácico.

El caso confirmado hace referencia a la persona que cumple con la definición operacional de sospechoso y que cuenta con diagnóstico confirmado por laboratorio emitido por el Instituto de Diagnóstico y Referencia Epidemiológicos (InDRE). ${ }^{\text {iv }}$

\section{LAS RECOMENDACIONES}

Considerando el riesgo que tienen los pacientes inmunosuprimidos de contraer SARS-CoV-2, la potencial demanda de servicios hospitalarios para la atención de pacientes infectados, así como el riesgo de infección de los profesionales de la salud, se recomienda la suspensión temporal de todos los programas de donación y trasplante de órganos y tejidos a nivel nacional hasta nuevo aviso. Solamente se mantendrá la realización de trasplantes en casos de urgencia nacional (hígado y corazón) y asignación prioritaria (riñón y córnea), que corresponden a situaciones que ponen en riesgo la vida y la calidad de la misma de los pacientes en lista de espera. Por lo tanto, lo anterior no implica una prohibición total de la actividad de donación y trasplantes.

En general, se ha acordado diferir los procedimientos quirúrgicos electivos (trasplantes de donador vivo y de donador fallecido no urgentes), para disminuir el riesgo de exposición a los pacientes y personal de la salud al SARS-CoV-2. En el caso de trasplante de tejido corneal, aunque no se puede hablar de un mayor riesgo en los pacientes postrasplante por la ausencia de inmunosupresión, múltiples instituciones internacionales como la Academia Americana de Oftalmología, la Sociedad Americana de Especialistas de Retina y el Colegio Americano de Cirujanos han acordado diferir también estos procedimientos.

\section{Trasplante en urgencias nacionales} y asignaciones prioritarias

Para realizar casos de urgencias de trasplante y asignaciones prioritarias, éstos deberán ser notificados al

En menores de cinco años de edad, la irritabilidad puede sustituir a la cefalea

iv El listado actualizado de laboratorios con reconocimiento por el InDRE, para realizar el diagnóstico de COVID-19 con fines de Vigilancia Epidemiológica se puede consultar en: https://www.gob.mx/salud/ documentos/aviso-epidemiologico-casos-de-infeccion-respiratoriaasociados-a-nuevo-coronavirus-2019-ncov
Registro Nacional de Trasplantes para su activación y aviso inmediato a las coordinaciones hospitalarias de donación, centros y consejos estatales de trasplantes y coordinaciones institucionales, y atender el caso lo más pronto posible.

Se recomienda considerar la oportuna participación de las unidades de vigilancia epidemiológica hospitalarias correspondientes en los comités internos de los hospitales, así como la comunicación con éstas y las jurisdicciones sanitarias para el seguimiento de los casos sospechosos o confirmados de COVID-19.

En estos casos se sugiere incluir, además de la aprobación por el Comité Interno de Trasplantes, la resolución por el Comité Hospitalario de Bioética y el consentimiento informado del receptor en el que se especifique la aceptación de riesgos en el contexto de la epidemia de COVID-19.

Para garantizar la seguridad de los receptores, se recomienda la inclusión de pruebas diagnósticas para COVID-19 como parte de los estudios pretrasplante en los casos de urgencia nacional y asignación prioritaria.

Donación de órganos y tejidos para

la atención de urgencias nacionales $\mathrm{y}$ asignaciones prioritarias

En caso de identificar a un potencial donador fallecido, el coordinador hospitalario de donación deberá contactar de inmediato al Centro Nacional de Trasplantes y consultar el Sistema Informático del Registro Nacional de Trasplantes (SIRNT) para verificar si existe alguna urgencia nacional o asignación prioritaria activa, y en caso afirmativo proceder con la donación.

Se recomienda la realización de pruebas diagnósticas de COVID-19 en todos los potenciales donantes para descartar la infección. Los establecimientos se deberán apoyar del Centro o Consejo Estatal de Trasplantes, así como la Coordinación Institucional correspondiente para gestionar la realización de estas pruebas.

Se deberá descartar la donación en los siguientes casos:

- Casos sospechosos o confirmados de COVID-19, hasta un periodo de tiempo de al menos 21 días desde la resolución de los síntomas.

- Pacientes sin datos clínicos compatibles, pero con resultado positivo en las pruebas diagnósticas.

- Prueba diagnóstica de COVID-19 no disponible. 


\section{Recomendaciones sobre receptores de trasplante}

Aunque hasta ahora no se cuenta con suficiente información del comportamiento del virus en pacientes con inmunodeficiencias primarias o secundarias, se consideran como grupo de alto riesgo los pacientes en tratamiento con inmunosupresores.

Se sugiere a los programas de trasplante establecer una línea de comunicación directa con los pacientes receptores de trasplante para la identificación y seguimiento de casos sospechosos o confirmados de COVID-19.

En caso de infección o sospecha, evitar que el paciente acuda al hospital, salvo en los casos de urgencia médica. Con la finalidad de garantizar la atención sin la necesidad de exponer al paciente, se recomienda a las instituciones implementar estrategias para que los familiares puedan acudir a las consultas y recibir los medicamentos, así como establecer una vía de comunicación para el seguimiento y atención no presencial del paciente.

En el documento también se incluye una serie de recomendaciones a los pacientes receptores y sus familias para que sean difundidas por los programas de trasplante, para disminuir el riesgo de contagio.

Reporte y seguimiento de receptores con COVID-19

Las recomendaciones también plantean el reporte al Centro Nacional de Trasplantes de los receptores de trasplantes de órganos y tejidos con COVID-19, toda vez que esta población, bajo tratamiento inmunosupresor en su mayoría, representa un grupo de riesgo importante para la presentación de esta enfermedad. A nivel internacional se han hecho esfuerzos para obtener la información de estos casos en receptores y se ha reportado la experiencia de múltiples centros. ${ }^{9,11,12,14,15}$

Para este fin, se definieron formatos de reporte y seguimiento de los pacientes detectados en esta situación, los cuales son llenados por los profesionales en los hospitales con la información del caso, y son enviados al CENATRA para su registro y análisis. En estos reportes se incluyen datos relativos a la identificación del paciente y su trasplante, inicio del cuadro clínico, confirmación de la infección, manejo de inmunosupresión y evolución del paciente, y en su caso la atención hospitalaria, especialmente el ingreso a la Unidad de Cuidados Intensivos (UCl) o intubación endotraqueal, como criterios de gravedad de la enfermedad.
La información se concentra en una base de datos, a la cual posteriormente tendrán acceso los responsables de programas y de las instituciones de salud, así como los integrantes de un grupo de trabajo para el análisis y desarrollo de recomendaciones clínicas específicas. El formato se encuentra alineado con el documento de reporte de casos en distintos países, lo cual facilitará el intercambio de información con los distintos sistemas y organismos nacionales.

El seguimiento de los pacientes también representa un reto para los hospitales durante esta crisis al ser más probable que se detecten los casos graves que requieren de hospitalización y no aquellos asintomáticos o cuadros leves. Detectar receptores posiblemente infectados o enfermos implica una búsqueda intencionada y un seguimiento más estrecho, para lo cual los hospitales han empezado a implementar una serie de estrategias de atención de manera remota.

Esta información se difunde semanalmente en el boletín informativo especial: Trasplantes y COVID-19, que contiene el resumen de la información obtenida. Este boletín representa la fuente oficial de consulta y divulgación de información en este tema para los profesionales y la ciudadanía, así como para las organizaciones nacionales e internacionales, y permitirá la retroalimentación de las acciones y toma de decisiones en el subsistema.

\section{LA APLICACIÓN DE LAS RECOMENDACIONES}

Con la finalidad de reforzar la comunicación y seguimiento del tema, se han llevado a cabo reuniones por videoconferencia para trasmitir la postura y recomendaciones sobre COVID-19 a grupos específicos como los centros, consejos estatales de trasplantes, coordinaciones institucionales de trasplantes, programas de trasplante de córnea y bancos de tejido ocular, gracias a la participación coordinada con sociedades científicas y asociaciones como la Sociedad de Trasplantes de América Latina y el Caribe (STALYC), Sociedad Mexicana de Trasplantes (SMT) y la Asociación Mexicana de Bancos de Tejidos Oculares (AMBTOC), así como de las instituciones de salud y hospitales tanto públicos como privados para transmitir y aplicar el mensaje de las recomendaciones.

Si bien se habla de una suspensión temporal de los programas, se conserva la posibilidad de atender casos de urgencia de trasplante o asignaciones prioritarias, como las fallas hepáticas fulminantes, choque cardiogénico o perforación corneal. Estos eventos que 
ponen en riesgo la vida o función del paciente de manera inmediata tienen una consideración especial en la evaluación del riesgo-beneficio de realizar un trasplante en esta crisis, y se han tomado las medidas necesarias para preservar este punto esencial.

El reto reside en garantizar la seguridad de los pacientes que se sometan a un trasplante en este periodo ante el riesgo de infección, pero también de los profesionales que intervienen en el proceso tanto de procuración como trasplante. Esta actividad frecuentemente implica el desplazamiento de profesionales a otras ciudades, vía aérea o terrestre, y la interacción cercana con profesionales y pacientes de áreas críticas como urgencias o terapia intensiva, por lo que se debe garantizar la disponibilidad de equipos de protección personal y protocolos de seguridad para llevar a cabo eventos de donación y trasplante de manera segura.

La realización de pruebas diagnósticas de COVID-19 en los donadores y receptores representa un reto para continuar con la actividad. Se han establecido canales de comunicación con las Secretarías de Salud Estatales a través de los centros y consejos estatales de trasplantes para facilitar la realización de las pruebas ante un eventual donador o caso de urgencia; sin embargo, el acceso puede no ser inmediato y el tiempo para obtener el resultado podría complicar la logística de la donación y el trasplante.

Probablemente la disponibilidad de pruebas pueda aumentar conforme avance la crisis y disminuya su demanda, facilitando la generación de donantes y, en consecuencia, los trasplantes. Se tendrá que contemplar también la inclusión de estas pruebas de forma rutinaria dentro de los protocolos a nivel nacional, tanto en donantes como en receptores, una vez pasada la pandemia. Esto representará el replanteamiento de muchos programas a nivel nacional y mundial para adaptarse a nuevos estándares de seguridad en este proceso.

La participación de los profesionales para la aplicación de las recomendaciones y la comunicación con los hospitales para identificar casos de urgencia ha permitido la atención de varios casos de asignación prioritaria de córnea, en colaboración interinstitucional para obtener los tejidos necesarios.

\section{CONCLUSIONES}

La pandemia por COVID-19 representa un antes y un después para los sistemas de salud a nivel mundial. El impacto en diversos programas, como donación y trasplantes, es innegable a múltiples niveles. Durante la crisis, se debe recordar que los donadores fallecidos «compiten» por un lugar en la terapia intensiva con los pacientes de COVID-19, y ante la disponibilidad de recursos y capacidad de respuesta se deben replantear las prioridades para hacer frente al problema. Tampoco se debe olvidar que un paciente postrasplante se considera población de riesgo para esta enfermedad, por lo que se debe adoptar una serie de medidas en los protocolos para minimizar este riesgo y garantizar la seguridad del paciente y profesionales.

La respuesta coordinada y efectiva del Subsistema Nacional de Donación y Trasplantes es necesaria para hacer frente a esta emergencia sanitaria, donde la colaboración entre las entidades federativas, las instituciones de salud y los sectores público y privado será determinante en los meses por venir. El CENATRA reitera su compromiso de mantener un seguimiento estrecho del tema y los avances que se vayan logrando para tomar las mejores decisiones de manera consensuada y coordinada, en beneficio siempre de los pacientes trasplantados y los que aún continúan en espera de la oportunidad de un trasplante, así como de la seguridad de los profesionales de la salud.

\section{REFERENCIAS}

1. van Doremalen $N$, Bushmaker $T$, Morris $\mathrm{DH}$, Holbrook MG, Gamble A, Williamson BN et al. Aerosol and surface stability of SARS-CoV-2 as compared with SARS-CoV-1. N Engl J Med. 2020; 382 (16): 1564-1567. doi: 10.1056/NEJMc2004973.

2. Sarukhan A. Un nuevo coronavirus, una nueva epidemia, muchas incógnitas. Published 2020. [Accessed April 17, 2020] Disponible en: https://www.isglobal.org/coronavirus.

3. Centers for Disease Control and Prevention. Severe outcomes among patients with coronavirus disease 2019 (COVID-19)United States, February 12-March 16, 2020. Morb Mortal Wkly Rep. 2020; 69 (12): 343-346. [Accessed April 17, 2020] Disponible en: https://www.cdc.gov/mmwr/volumes/69/wr/ mm6912e2.htm.

4. Centros para el Control y la Prevención de Enfermedades CN de $V$ y ER (NCIRD). Marco de intervalos de una pandemia (PIF). Influenza pandémica (influenza). CDC. Published 2016. [Accessed April 17, 2020] Disponible en: https://espanol. cdc.gov/flu/pandemic-resources/national-strategy/intervalsframework.html.

5. World Health Organization. Situation report-85 highlights. Geneva; 2020. [Accessed April 17, 2020] Disponible en: https://www.who.int/teams/risk-communication/infodemicmanagement.

6. Pandemia de enfermedad por coronavirus de 2020 en México - Wikipedia, la enciclopedia libre. [Accessed April 17, 2020] Disponible en: https://es.wikipedia.org/wiki/Pandemia_de_ enfermedad_por_coronavirus_de_2020_en_México\#cite_ note-3.

7. Consejo de Salubridad General declara emergencia sanitaria nacional a epidemia por coronavirus COVID-19. Secretaría de 
Salud. [Accessed April 17, 2020] Disponible en: https://www. gob.mx/salud/prensa/consejo-de-salubridad-general-declaraemergencia-sanitaria-nacional-a-epidemia-por-coronaviruscovid-19-239301.

8. Ning L, Liu L, Li W, Liu H, Wang J, Yao Z et al. Novel coronavirus (SARS-CoV-2) infection in a renal transplant recipient: Case report. Am J Transplant. 2020. doi: 10.1111/ajt.15897. [Epub ahead of print]

9. Guillen E, Pineiro GJ, Revuelta I, Rodriguez D, Bodro M, Moreno A et al. Case report of COVID-19 in a kidney transplant recipient: Does immunosuppression alter the clinical presentation? Am J Transplant. 2020. doi: 10.1111/ajt.15874. [Epub ahead of print]

10. Aslam S, Mehra MR. COVID-19: Yet another coronavirus challenge in transplantation. J Heart Lung Transplant. 2020; 39 (5): 408-409. doi: 10.1016/j.healun.2020.03.007.

11. Li F, Cai J, Dong N. First cases of COVID-19 in heart transplantation from China. J Heart Lung Transplant. 2020; 39 (5): 496-497. doi: 10.1016/j.healun.2020.03.006.

12. Zhu L, Xu X, Ma K, Yang J, Guan H, Chen S et al. Successful recovery of COVID-19 pneumonia in a renal transplant recipient with long-term immunosuppression. Am J Transplant. 2020. doi: 10.1111/ajt.15869. [Epub ahead of print]
13. Michaels MG, La Hoz RM, Danziger-Isakov L, Blumberg EA, Kumar D, Green M et al. Coronavirus disease 2019: Implications of emerging infections for transplantation. Am J Transplant. 2020. doi: 10.1111/ajt.15832. [Epub ahead of print]

14. Coates PT, Wong G, Drueke T, Rovin B, Ronco P; Associate Editors, for the Entire Editorial Team. Early experience with COVID-19 in kidney transplantation. Kidney Int. 2020. pii: S00852538(20)30362-8. doi: 10.1016/j.kint.2020.04.001. [Epub ahead of print]

15. Banerjee D, Popoola J, Shah S, Ster IC, Quan V, Phanish M. Kidney Int. COVID-19 infection in kidney transplant recipients. 2020. pii: S0085-2538(20)30361-6. doi: 10.1016/j. kint.2020.03.018. [Epub ahead of print]

\section{Correspondencia:}

Dr. José André Madrigal-Bustamante

Carr. Picacho-Ajusco Núm. 154,

Jardines en la Montaña, 14210,

Tlalpan, Ciudad de México.

Tel: 54879902, ext. 51406

E-mail: jose.madrigal@salud.gob.mx 